REVIEW ARTICLE

DOI: $10.1038 / s 41467-017-01049-7$

\title{
The emergence of piRNAs against transposon invasion to preserve mammalian genome integrity
}

\author{
Christina Ernst (1D ${ }^{1}$, Duncan T. Odom (iD ${ }^{1} \&$ Claudia Kutter ${ }^{2}$
}

Transposable elements (TEs) contribute to the large amount of repetitive sequences in mammalian genomes and have been linked to species-specific genome innovations by rewiring regulatory circuitries. However, organisms need to restrict TE activity to ensure genome integrity, especially in germline cells to protect the transmission of genetic information to the next generation. This review features our current understandings of mammalian PIWI-interacting RNAs (piRNAs) and their role in TE regulation in spermatogenesis. Here we discuss functional implication and explore additional molecular mechanisms that inhibit transposon activity and altogether illustrate the paradoxical arms race between genome evolution and stability.

$\mathrm{R}$ epetitive sequences make up a large proportion of mammalian genomes, with up to two thirds of the human genome being repeat-derived ${ }^{1}$. A subfraction of these repeats are mobile elements, also termed transposable elements (TEs), that play crucial roles in driving genome evolution by fueling the development of new genes, introducing novel immune strategies such as $\mathrm{V}(\mathrm{D}) \mathrm{J}$ recombination, and rewiring gene regulatory circuitries ${ }^{2}$. However, such plasticity comes at the price of causing potential deleterious effects as a result of uncontrolled retrotransposition and therefore requires a tight handling of TEs by their host cells ${ }^{3}$. This is especially important in cells contributing to the germline, to ensure the integrity of the genome that is passed on to the next generation. To this effect, an additional layer of retrotransposon control has evolved in the metazoan germline that is based on small RNA-mediated recognition of TE transcripts called the piRNA pathway.

Active retrotransposition is more frequent in germ cells since the epigenetic reprogramming that primes these cells for totipotency also results in the derepression of TEs ${ }^{4}$. A requirement for the successful retrotransposition of a TE is active cell divisions ${ }^{5}$. Hence, the burden is heavier during male germ cell development in mammals, which is marked by continuous waves of spermatogenesis throughout the life span, compared to female germ cells of which a defined number arrests in meiosis I during embryonic development and only matures after the onset of sexual maturity ${ }^{6}$.

In this review, we will discuss the role of PIWI-interacting RNAs (piRNAs) throughout mammalian, mostly mouse, spermatogenesis and their interplay with transposable elements and briefly touch on additional silencing mechanisms controlling the activity of transposable elements.

\footnotetext{
${ }^{1}$ University of Cambridge, Cancer Research UK Cambridge Institute, Robinson Way, Cambridge CB2 ORE, UK. ${ }^{2}$ Department of Microbiology, Tumor and Cell Biology, Science for Life Laboratory, Karolinska Institute, Nobels väg 16, 17177 Stockholm, Sweden. Correspondence and requests for materials should be addressed to C.K. (email: claudia.kutter@ki.se)
} 
a

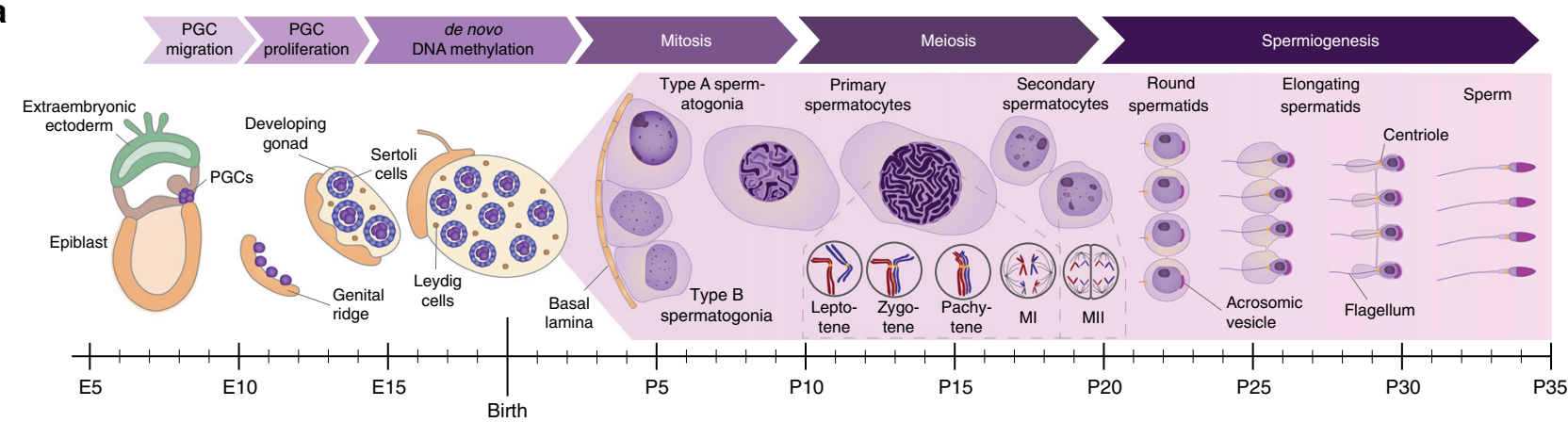

b
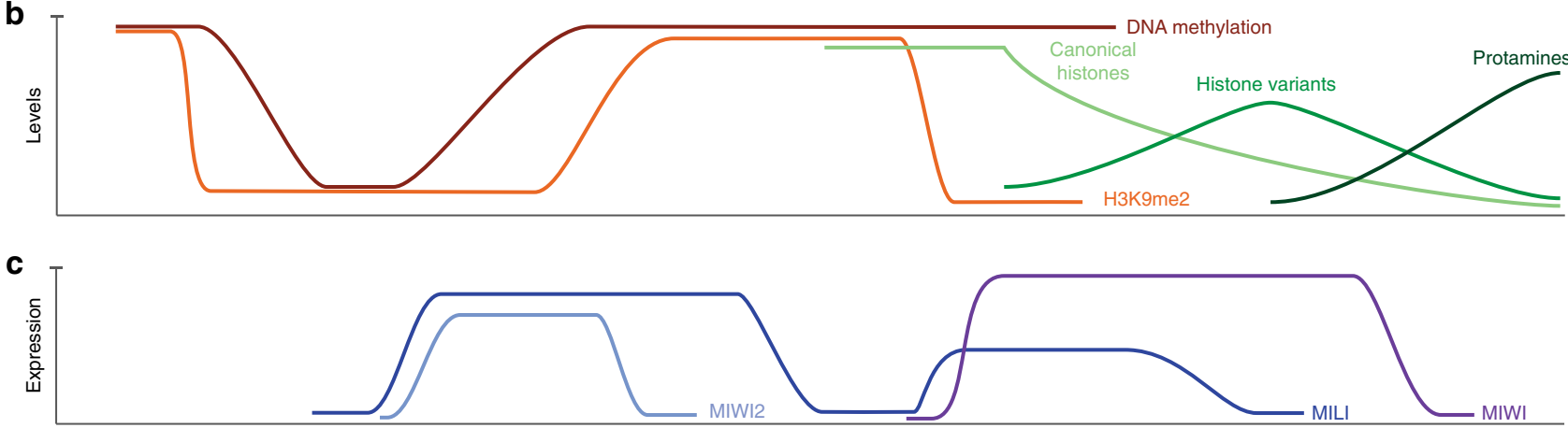

Fig. 1 Male germ cell nomenclature and developmental dynamics of mouse spermatogenesis. a Gametogenesis starts during embryonic development when primordial germ cells (PGCs) are defined and migrate to the genital ridge to form the gonads. Spermatogenesis initiates shortly after birth in synchronized waves. At 10 days post birth (P10), spermatogonial stem cells differentiate into primary spermatocytes that are committed to undergo meiosis. Two consecutive cell divisions (meiosis I and II (MI and MII)) without an intermediate S-phase result in the production of haploid gametes that are called round spermatids. These cells can be found as early as P20 and then undergo spermiogenesis during which the cells elongate and develop sperm-specific structures such as the acrosome and the flagellum to form mature sperm cells. $\mathbf{b}$ The process of gametogenesis is associated with extensive epigenetic reprogramming accompanied by drastic changes in DNA methylation and histone modifications such as H3K9me2. During later stages of spermatogenesis, global changes in histone composition and finally a histone-to-protamine exchange result in chromatin compaction. c The three PIWI proteins encoded in the mouse genome show very specific expression profiles throughout spermatogenesis and reflect functionally distinct aspects of the piRNA pathway at different stages of spermatogenesis

\section{Regulatory dynamics of mouse spermatogenesis}

Gametogenesis is a complex process that starts as early as embryonic day 7.5 (E7.5) with the emergence of primordial germ cells (PGCs) that migrate to and populate the genital ridges at E10.5-E11.5 (Fig. 1a). Migratory PGCs experience various epigenetic changes, such as global erasure of histone $\mathrm{H} 3 \mathrm{~K} 9 \mathrm{me} 1 / 2$, which is linked to decreased expression of the H3K9 methyltransferase G9a-like protein, as well as an increase in H3K27me3 and various histone variants (Fig. 1b) ${ }^{7}$. PGCs arrive at the genital ridge during midgestation at E10.5-11.5, where they continue their reprogramming resulting in a global loss of DNA methylation ${ }^{8}$. Once PGCs are residing within the gonads, sexual dimorphism occurs around E11.5 and male PGCs continue to proliferate in the gonads until they enter mitotic arrest at E14 (at which point $\sim 25.000$ PGCs are found in each gonad) $)^{9}$. Male PGCs remain mitotically arrested until postnatal day 2 (P2), during which time de novo DNA methylation and the establishment of paternal imprints takes place ${ }^{10}$. This process is mediated by the de novo DNA methyltransferases DNMT3A and DNMT3B as well as their catalytically inactive interaction partner DNMT3L ${ }^{11}$. DNMT3L is essential for spermatogenesis by guiding de novo DNA methylation and binding to unmethylated histone H3 lysine 4 tails $^{12}$.

Male PGCs resume cell division shortly after birth and give rise to type-A spermatogonia, which are found on the basement membrane of seminiferous tubules. These cells have self-renewing potential but also produce type-B spermatogonia, which can enter meiosis and become spermatocytes ${ }^{13}$. After several rounds of mitotic divisions from P2 onwards, the first meiosis is initiated in a synchronous manner around P10. As cells progress through spermatogenesis, they move away from the basement membrane toward the lumen of seminiferous tubules and enter pre-leptotene/leptotene, zygotene, pachytene, and diplotene stages on P10, 12,14 , and 17 , respectively ${ }^{13}$. Prophase of meiosis I is unique in that it promotes transfer of genetic information between the parental genomes via crossovers. To allow meiotic crossovers, homologous chromosomes synapse during the zygotene stage and form bivalents ${ }^{14}$. This is mediated by DNA double-strand breaks (DSBs) that are specifically induced by SPO $11^{15}$ and enable homologous recombination (HR) between non-sister chromatids of homologous chromosomes. Crossovers are essential for proper chromosome synapsis and occur in hotspots across mammalian genomes, directed by PRDM9 ${ }^{16-18}$. Failures in de novo DNA methylation have been shown to cause mislocalization of crossovers resulting in spermatogenic arrest at the pachytene stage ${ }^{19}$. When reaching the diplotene stage of prophase I, homologous chromosomes begin to separate but are still attached to each other, thereby forming the characteristic structures called chiasmata. Eventually, bivalents are arranged on the metaphase plate and homologous chromosomes are separated to opposite poles followed by cytokinesis ending the first meiotic division.

The second meiotic division follows promptly and generates the first set of round spermatids to be found at P20. Round spermatids then undergo a complex differentiation program called spermiogenesis, during which they form sperm-specific 


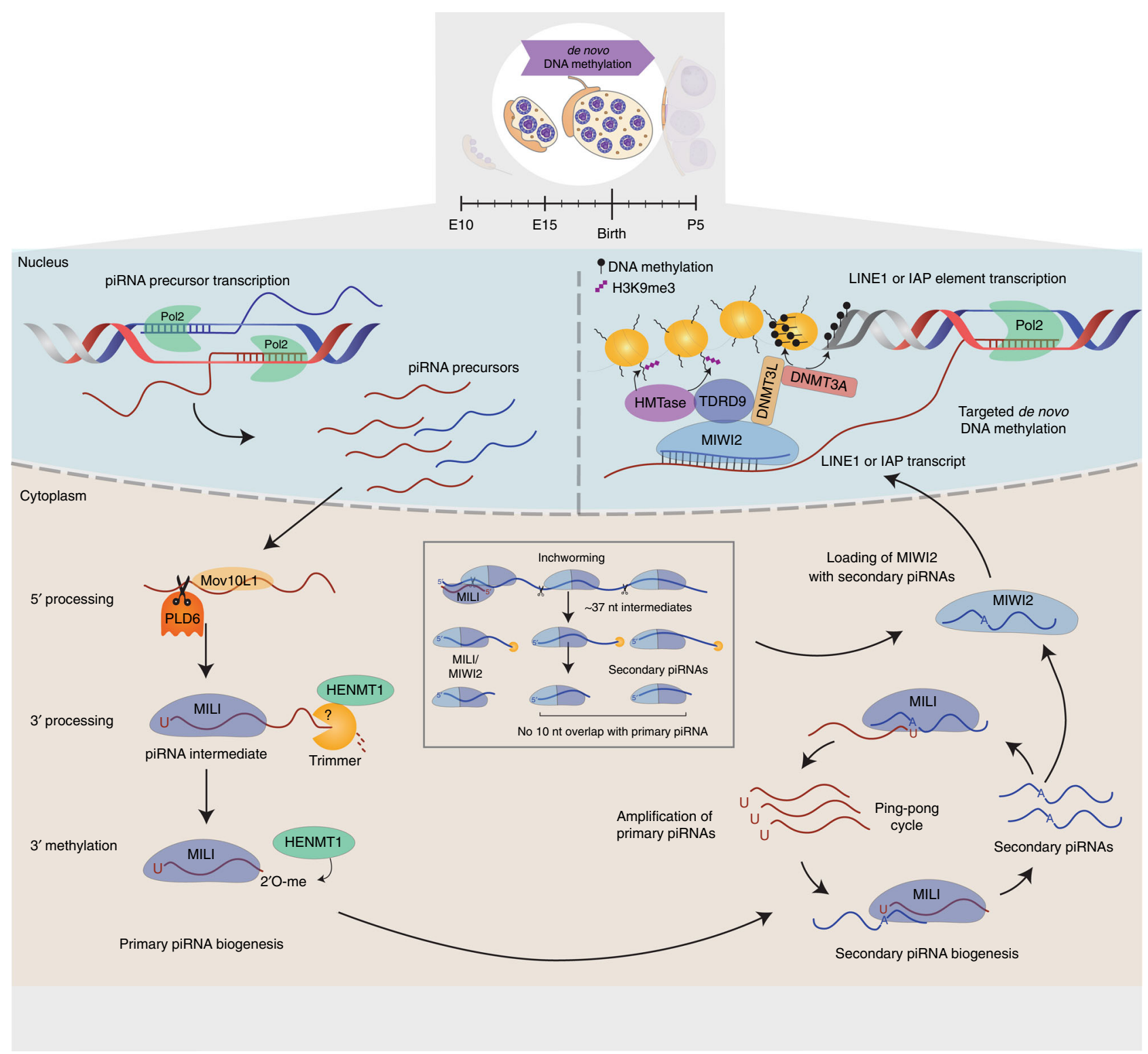

Fig. 2 Biogenesis and function of pre-pachytene piRNAs in the mouse male germline. Pre-pachytene piRNAs are expressed during embryonic development from genomic loci that often display bidirectional transcription. piRNA precursors are exported to the cytoplasm and directed to the processing machinery. After 5' end processing by PLD6, the piRNA intermediates associate with MILI. The 3' ends of piRNA intermediates are then trimmed and 3' methylated by Trimmer and HENMT1, respectively, to generate mature primary piRNAs bound to MILI. These complexes can then engage in the ping-pong cycle leading to the cleavage of complementary antisense transcripts and the production of secondary piRNAs bound to either MILI or MIWI2. Secondary piRNAs are characterized by a $10 \mathrm{nt}$ overlap in their $5^{\prime}$ ends with primary piRNAs as a result of MILI-directed cleavage. Loading of MIWI2 with secondary piRNAs induces its translocation into the nucleus, where it functions in the transcriptional silencing of transposable elements (TEs). This involves the recruitment of the de novo DNA methylation machinery (DNMTs) as well as histone methyltransferases (HMTase) resulting in targeted DNA methylation and H3K9me3mediated heterochromatinization of TE loci, such as LINEs and IAPs. An additional piRNA biogenesis mechanism, called inchworming, can produce MILIand MIWI2-associated piRNAs from primary piRNA precursors without the characteristic 10 nt overlap with other piRNA species

structures such as the acrosome and flagellum and undergo nuclear compaction ${ }^{20}$. The latter is achieved via genome-wide replacement of histones with transition proteins at first, followed by protamines, which results in up to a 20 times more condensed structure due to the basic character of these proteins ${ }^{21}$. To allow the transition, a loose chromatin structure is adopted and mediated in part by the incorporation of various testis-specific histone variants, which leads to a global transcriptional activation including TEs ${ }^{22-24}$. Histone replacement, however, was shown to be incomplete with some genomic regions retaining their histones including epigenetic modifications to potentially prime these regions for early transcription after fertilization ${ }^{25-27}$.
The different waves of epigenetic reprogramming and global remodeling of the genome allow repetitive elements to become transcriptionally active in the male germline at several developmental time points. To prevent these repetitive elements from causing genome instability, additional control mechanisms are required during spermatogenesis.

\section{Mouse PIWI proteins}

The mouse male germline expresses three proteins of the $\mathrm{P}$ element-induced wimpy testis (PIWI) family: MILI, MIWI, and MIWI2, all of which are essential for spermatogenesis and male 
fertility $^{28-30}$. Each PIWI protein exhibits a unique expression profile throughout spermatogenesis (Fig. 1c) and associates with a defined population of small non-coding RNAs called PIWIinteracting RNAs (piRNAs) ${ }^{31-34}$. PIWI proteins are members of the highly conserved Argonaute family and are characterized by their PIWI, MID and PAZ domains ${ }^{35}$. The PIWI domain is essential for the endonucleolytic slicer activity of PIWI proteins ${ }^{36}$, while the MID and PAZ domains function in anchoring the $5^{\prime}$ and $3^{\prime}$ ends of piRNAs, respectively ${ }^{37-39}$. In mice, PIWI proteins and piRNAs are almost exclusively found in the male germline, with low expression levels of MILI and piRNAs in oocytes ${ }^{30,40,41}$ and some recent reports indicating their expression in somatic cells ${ }^{42,} 43$

\section{Primary piRNA biogenesis}

Besides their association with different PIWI proteins, piRNAs are further distinguished based on their biogenesis. The biogenesis of primary piRNAs begins with the transcription of a single-stranded piRNA precursor molecule by RNA polymerase II (Pol II) from defined genomic locations termed piRNA clusters (Fig. 2). piRNA precursor molecules are then exported into the cytoplasm, which might be facilitated by Maelstrom (MAEL), a conserved HMGbox domain protein with RNA-binding activity ${ }^{44}$. Once in the cytoplasm, piRNA precursors associate with the RNA helicase MOV10L1, whose ATP-dependent unwinding activity is essential for piRNA biogenesis as illustrated by the increase in piRNA precursor transcripts and almost complete lack of mature piRNAs in MOV10L1 knockouts, or catalytically defective mutants ${ }^{45}, 46$. MOV10L1 is believed to fuel piRNA precursors into the biogenesis pathway by facilitating the interaction with PLD6 (MITOPLD), the endonuclease that generates the $5^{\prime}$ end of primary piRNAs ${ }^{47,48}$. Intermediate fragments with a $5^{\prime}$ uridine (1U) are preferentially bound by PIWI proteins and thereby stabilized, leading to the strong $1 \mathrm{U}$ bias that is characteristic for primary piRNAs ${ }^{49}$. PIWI-bound piRNA intermediates then undergo $3^{\prime}$ end processing, which most likely involves exonucleolytic shortening by a $3^{\prime}-5^{\prime}$ trimmer that has not been identified in mammals yet. The process is facilitated by Tdrkh, a Tudor and $\mathrm{KH}$ domaincontaining protein, whose absence causes the accumulation of 31-37 nucleotide (nt) long intermediates ${ }^{50}$. The final length of piRNAs during $3^{\prime}$ end maturation is determined by the footprint of their associated PIWI protein, which protects the $3^{\prime}$ end from further trimming ${ }^{51}$. This generates size-specific piRNA populations for the different PIWI proteins with a mean length of $26 \mathrm{nt}$ for MILI-, $28 \mathrm{nt}$ for MIWI2-, and $30 \mathrm{nt}$ for MIWI-bound piRNAs ${ }^{32}, 52,53$. Mature $3^{\prime}$ ends are then $2^{\prime}-\mathrm{O}$ methylated by the RNA methyltransferase HENMT1 resulting in increased stability and binding to the PAZ domain of their PIWI protein ${ }^{54}$.

\section{Secondary piRNA biogenesis}

Primary piRNAs can then guide the PIWI-mediated cleavage of antisense transcripts between nucleotide 10 and 11 to produce secondary piRNAs, a process known as the ping-pong cycle. Secondary piRNAs therefore have a defining $10 \mathrm{nt} 5^{\prime}$ overlap with the primary piRNA that mediated the cleavage and often have adenine at their tenth position $(10 \mathrm{~A})^{53}$. This $10 \mathrm{~A}$ bias has long been thought to originate from complementarity to the $5^{\prime}$ uridine of primary piRNAs. However, base pairing at the first position is not necessary for efficient cleavage by Argonaute proteins ${ }^{55}$ and it appears that mammalian PIWI proteins rather have a preference for adenine at the $\mathrm{t} 1$ position that is not determined by base pairing ${ }^{56}$. Secondary piRNAs in turn can then fuel the production of the initiating primary piRNAs, thereby completing the pingpong cycle. In the mouse, secondary piRNA production is driven by the slicer activity of MILI and most ping-pong cycles rely on homotypic MILI:MILI interactions with occasional heterotypic MILI:MIWI2 associations ${ }^{57}$. These heterotypic ping-pong interactions are however not reciprocal and the loading of MIWI2 with secondary piRNA is entirely dependent on MILI ${ }^{53}$. The disengagement of MIWI from the ping-pong cycle was shown to be due to active repression of the ping-pong cycle by RNF17, a Tudor family member, in meiotic cells. RNF17 knockout demonstrated that MILI:MIWI ping-pong interactions can occur in vivo, but result in spermatogenic arrest due to ping-ponginitiated piRNA responses against protein-coding genes ${ }^{58}$.

\section{Inchworm piRNA biogenesis}

In addition to the primary and secondary piRNA biogenesis pathways, a separate mechanism termed "inchworming" fuels the production of MIWI2-associated piRNAs. Inchworming is initiated by MILI-loaded piRNAs that direct slicing of a target transcript. Instead of giving rise to only one conventional secondary piRNA, multiple evenly spaced, $37 \mathrm{nt}$ long piRNA intermediates are formed in $5^{\prime}-3^{\prime}$ direction from the initial cleavage product. These intermediates can associate with both MILI and MIWI2 and are trimmed at their $3^{\prime}$ ends to the typical length of 26 and $28 \mathrm{nt}$, respectively. Inchworm piRNAs do not have the characteristic $10 \mathrm{nt}$ overlap of secondary piRNAs but display a strong $1 \mathrm{U}$ bias $^{59}$.

MILI-associated piRNAs that initiate inchworming are in sense orientation to L1 elements and therefore lead to the production of large amounts of antisense inchworm piRNAs in contrast to the piRNAs produced in the ping-pong cycle. Thus, inchworming presents an amplification mechanism for antisense piRNAs that can silence L1 elements on the transcriptional or posttranscriptional level by associating with either MIWI2 or MILI, respectively ${ }^{59}$.

\section{Pre-pachytene piRNAs regulate DNA de novo methylation}

MILI is the first PIWI protein to be expressed during development, starting at E13.5 in PGCs with a dynamic expression profile up until the round spermatid stage ${ }^{30,60,61}$. In contrast, MIWI2 is only expressed during a short period of time, starting at E14.5 until shortly after birth with protein levels being undetectable by $\mathrm{P} 4^{53}, 61$. This expression window largely coincides with mitotic arrest of PGCs and de novo DNA methylation. Knockout of either MILI or MIWI2 leads to upregulation of TEs resulting in a spermatogenic arrest; however, not during the time of their joint expression, but only several days and cell divisions later in early pachytene spermatocytes ${ }^{62}$. piRNAs expressed during this period are termed pre-pachytene piRNAs and are characterized by a high occurrence of TEs in sense orientation, suggesting that most primary pre-pachytene piRNAs derive directly from TE transcripts ${ }^{52,53}$. These piRNAs associate with MILI and drive the production of secondary piRNAs through recognition of antisense transcripts, which then associate with either MILI or MIWI2. This amplification method results in fine-tuning of the piRNA population and specific enrichment of those piRNAs that target the most active TEs.

While MILI is found exclusively in the cytoplasm in perinuclear granules called pi-bodies, MIWI2 is predominantly found in the nucleus but also localizes to cytoplasmic granules in the vicinity of pi-bodies, termed piP-bodies ${ }^{63}$. Translocation of MIWI2 into the nucleus is however dependent on its association with piRNAs and therefore on the slicer activity of MILI that is necessary to load MIWI2 with secondary piRNAs ${ }^{53}$. In contrast, the slicer activity of MIWI2 itself is dispensable for spermatogenesis, as catalytically inactive MIWI2 mutants show normal fertility and only modest upregulation of $\mathrm{TEs}^{57}$. This strongly suggests that MIWI2's essential function during spermatogenesis 
defers from post-transcriptional gene silencing (PTGS) of TE transcripts in the cytoplasm and is rather related to its nuclear function. Indeed, MIWI2 is required for the establishment of de novo DNA methylation over TEs acting upstream of DNMT3L in $\mathrm{PGCs}^{53}$ (Fig. 2).

De novo methylation happens in two waves and many TEs manage to escape the first "generic" wave that occurs by E16.5. Interestingly, many of these evading TEs (mainly long interspersed element 1 (LINE1s) and intracisternal particle A (IAPs)) correspond to evolutionary young elements that also become activated as a result of MILI knockout and consequently unloaded MIWI ${ }^{64}$. MIWI2 is believed to use antisense piRNAs as guides to detect nascent TE transcripts in the nucleus and recruits not only the de novo DNA methylation machinery ${ }^{65}$, but also histone methyltransferase to establish a repressive chromatin landscape especially across LINE1 elements ${ }^{66}$. Interestingly, preferential targeting of LINE1 elements by MIWI2 was also observed for DNA methylation. While loss of MIWI2 results in demethylation and derepression of mostly LINE1 elements, MILI deficiency causes DNA methylation defects across a wider range of TE families, including both LINE1 and LTR elements. This suggests that MILI can induce DNA methylation in a MIWI2-independent manner, however the underlying mechanisms are unknown ${ }^{67,68}$. In contrast to these observations, a recent report showed greater disruption of DNA remethylation upon loss of Miwi2 compared to Mili, suggesting a MILI-independent piRNA biogenesis pathway in fetal male gonads ${ }^{69}$.

As mentioned above, spermatogenic arrest in MILI, MIWI2, or DNMT3L mutants does not occur until the pachytene stage but is characterized by a global derepression of LINE1 and IAP elements. It thus appears that DNA methylation is dispensable for TE silencing until the onset of meiosis due to additional transcriptional silencing mediated by $\mathrm{H} 3 \mathrm{~K} 9 \mathrm{me} 2^{60}$. This repressive histone mark, however, displays a dynamic pattern during spermatogenesis and completely disappears at the pachytene stage (Fig. 1b) ${ }^{19}$. Loss of $\mathrm{H} 3 \mathrm{~K} 9 \mathrm{me} 2$ can be correlated with the expression of the histone methyltransferase G9a, which disappears at the leptotene stage preceding the $\mathrm{H} 3 \mathrm{~K} 9 \mathrm{me} 2 \operatorname{loss}^{70}$. In DNA methylation mutants, the loss of repressive histone marks is concomitant with the gain of active marks such as H3K4me3. This results in the aberrant transcriptional activation of several classes of young repeat elements leading to the spermatogenic arrest at the pachytene stage ${ }^{19}$. Changes in the chromatin landscape of TEs perturb the meiotic process by relocalizing SPO11-mediated DSBs to repetitive elements. It is currently unclear whether the induced apoptosis is due to potential HR between non-allelic positions when repetitive elements participate in synapsis, or whether overall synapsis and repair is impaired due to a sequestration of the meiotic recombination machinery away from canonical recombination hotspots. Regardless of the underlying cause, this shows that even though spermatogenic arrest occurs several days and cell replications after the loss of DNA methylation, meiosis is sensitive to the transcriptional activation of TEs as these can disturb the dynamics of meiotic recombination. This appears to be a safeguard mechanism to eliminate cells in which de novo methylation of TEs has not been sufficient during reprogramming of PGCs. Beyond transposon control, pre-pachytene piRNAs have been implicated in the establishment of paternal imprinting by directing de novo DNA methylation to the differentially methylated region of the Rasgrf1 locus ${ }^{71}$.

Once MIWI2 expression ceases, MILI is the only PIWI protein expressed in male germ cells with its own expression levels decreasing gradually to very low or undetectable levels in pre-leptotene and leptotene spermatocytes until it increases again during the zygotene to pachytene transition ${ }^{60}$. Pre-pachytene
piRNAs that engage with MILI during this time are involved in PTGS of TE transcripts that escape transcriptional gene silencing. This is supported by observations from conditional MILI knockout mice, in which the protein or its catalytic activity was removed at the differentiating spermatogonia stage. Despite intact DNA methylation in these animals, loss of MILI nevertheless results in spermatogenic arrest and high apoptosis ${ }^{60}$. Based on the lack of PIWI protein expression, it is suggested that pre-leptotene to late zygotene spermatocytes do not have an active piRNA pathway. This might be necessary to deplete the pool of prepachytene piRNAs from these cells to free up MILI proteins to engage with the new class of piRNAs about to be expressed.

\section{Pachytene piRNAs regulate TEs post transcriptionally}

As the first wave of spermatocytes reaches the pachytene stage of meiosis, the transcription of the third PIWI family member MIWI starts together with a new class of piRNAs termed pachytene piRNAs ${ }^{32-34}$. Pachytene piRNAs are transcribed from large intergenic non-coding and $3^{\prime}$ untranslated regions of protein-coding gene $\operatorname{loc}^{39,}, 2$, which have no unifying genomic sequence or motif definition and are fast evolving across mammals ${ }^{73}$. The majority of pachytene piRNAs $(>80 \%)$ map uniquely to the genome, thereby allowing unambiguous identification of piRNA clusters ${ }^{32}$. Pachytene piRNA clusters can be either uni- or bidirectionally transcribed. Although there is a fewer number of bidirectional clusters annotated in the genome, they contribute to the majority of pachytene piRNAs (Fig. 3). Transcription of these clusters is facilitated by the ancestral transcription factor A-MYB. The resulting piRNA transcripts are $5^{\prime}$ capped and polyadenylated. However, A-MYB is not specific to piRNA clusters as it also drives its own expression as well as other members of the piRNA pathway in a positive feed-forward loop and thus cannot be the sole determining factor that distinguishes piRNA cluster transcripts from conventional mRNAs ${ }^{74}$. This was further supported by a recent study showing that the deletion of an A-MYB-binding site (GACAGTTA) from the promoter of a pachytene piRNA cluster resulted in a reduction but not complete depletion of piRNA precursor transcript levels and processed pachytene piRNAs ${ }^{75}$. This suggests that what directs piRNA precursors into the biogenesis pathway is more likely to be an RNA-based recognition signal and could involve the formation of G-quadruplex structures ${ }^{45}$. Similar to the observations in fetal gonocytes $^{63}$, the piRNA pathway components involved in the processing and function of pachytene piRNAs also show distinct localization patterns to cytoplasmic nuage structures termed chromatoid bodies in mid-to-late meiotic spermatocytes and haploid spermatids ${ }^{76,77}$.

In contrast to pre-pachytene piRNAs, pachytene piRNAs have a surprisingly low repeat content $(<20 \%)^{32}$, that is in fact lower than the genome average ${ }^{78}$, thereby calling their importance in the regulation of TEs into question. However, it has been demonstrated that MIWI's slicer activity is necessary for the accurate silencing of LINE1 elements in spermatocytes ${ }^{79}$. Nevertheless, the functional division between repeat-targeting and non-repeatassociated pachytene piRNAs still remains unclear.

Pachytene piRNA clusters have been shown to produce novel artificial piRNA sequences upon introduction of transgenic sequences $^{80}$. A similar scenario is possible for new TE insertions, which has led to the suggestion that piRNA clusters may serve as "genomic sinks" to capture active TEs ${ }^{44,81}$. However, in contrast to observations in Drosophila that show higher TE insertion rates into piRNA clusters ${ }^{82}$, no such bias has been described in mammals so far. It is therefore still unclear how the pachytene piRNA system adapts to newly invading repetitive elements and how quickly a new piRNA response can be installed. 


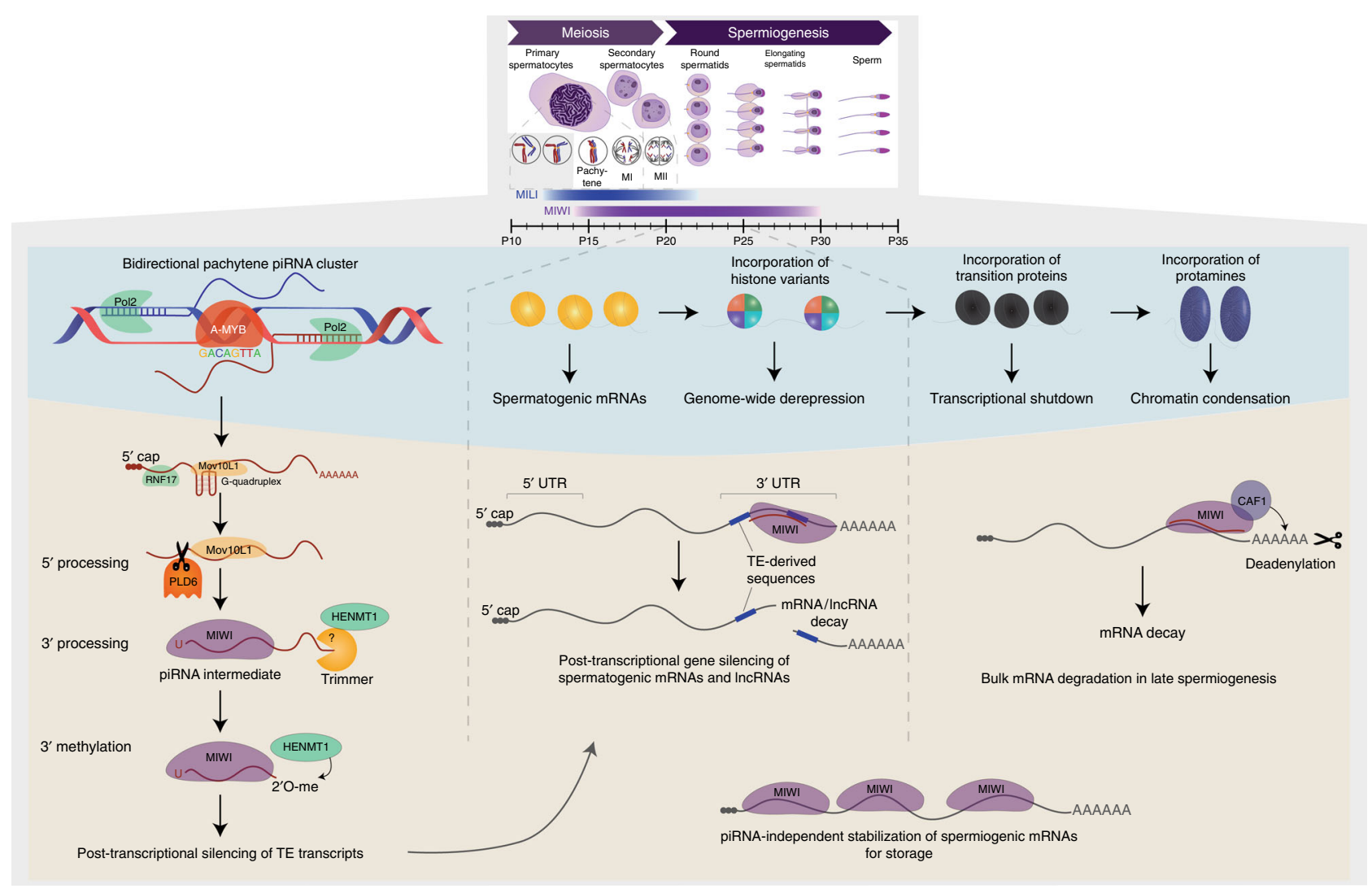

Fig. 3 Multiple roles of action for pachytene piRNAs during mouse spermatogenesis. Pachytene piRNA expression starts during prophase of the first meiotic division when spermatocytes reach the pachytene stage. This expression is orchestrated by the transcription factor A-MYB, which drives expression of piRNA clusters and other piRNA pathway-related genes. Pachytene piRNAs are transcribed from distinct clusters in the genome often carrying bidirectional promoters. piRNA precursors are then processed in the same fashion as pre-pachytene piRNAs and mature pachytene piRNAs associate with either MILI or MIWI. Pachytene piRNAs engage in a myriad of functions throughout spermatogenesis, including the post-transcriptional silencing of TE transcripts but also non-TE-related functions. After meiosis, round spermatids undergo extensive epigenetic remodeling, resulting in a genome-wide derepression due to the incorporation of histone variants, followed by transition proteins that lead to a transcriptional shutdown, and finally replacement of histones with protamines leading to chromatin condensation. During this differentiation process, pachytene piRNAs regulate spermatogenic mRNAs and IncRNAs that become transcribed due to the genome-wide derepression. Furthermore, MIWI is involved in the piRNA-independent stabilization of spermiogenic mRNAs to allow storage and translation after the transcriptional shutdown. Toward later stages, pachytene piRNAs direct global mRNA degradation in association with MIWI, which recruits CAF1 to induce deadenylation resulting in mRNA decay

\section{Non-repeat-related functions of pachytene piRNAs}

MIWI remains actively transcribed until the round spermatid stage and is the only PIWI protein detected in late round and elongating spermatids ${ }^{29}$. These late stages of spermatogenesis are associated with major epigenetic changes and various functions have been implicated for non-repeat-related pachytene piRNAs. For example, the transitioning from histone- to protamine-bound DNA in round spermatids requires open and accessible chromatin, which leads to a genome-wide loosening of transcriptional control and the expression of spurious transcripts including many long non-coding RNAs (lncRNAs) ${ }^{23}, 83$. Pachytene piRNAs have been shown to be essential in targeting many of these mRNA and IncRNAs to regulate their expression via PTGS $^{84}$ (Fig. 3). Interestingly, mutant mice for HENMT1, which lack methylation of piRNA $3^{\prime}$ ends leading to piRNA instability, exhibit a global loss of piRNAs resulting in the deregulation of meiotic transcripts ${ }^{54}$, further supporting the notion that piRNAs play a role in targeting and regulating meiotic gene expression on a post-transcriptional level ${ }^{85}$.

Recent work cloning a human piRNA cluster into the mouse and identifying mRNAs that are targeted by human ectopic piRNAs, shed light on the target specificity of this mechanism ${ }^{86}$. For a subset of ectopic piRNA sequences that overlapped with cleavage products profiled via $5^{\prime}$ RACE (Rapid amplification of cDNA ends), a perfect match between nucleotides $2-11$ with a maximum of four mismatches between nucleotides $12-21$ was detected. In support of this model, the observed male sterility phenotype of this mouse was explained by silencing of the germline-specific gene Dpy19L2 by one of the ectopically expressed human piRNAs. Double transgenic mice, expressing human piRNAs as well as the human Dpy19L2 gene that does not contain a target site for human piRNAs, were indeed fertile and able to produce offspring 86 .

During later stages of spermatogenesis in elongating spermatids, pachytene piRNAs were shown to direct the elimination of mRNAs. This was independent of the slicer activity of MIWI but required interaction with the deadenylase CAF1 to induce deadenylation and subsequent degradation of mRNAs ${ }^{87}$. Further functional implications for MIWI were made that are independent of associated piRNAs. In that case, MIWI binds to and stabilizes spermiogenic mRNAs that are stored for translation at later time points when transcription has been switched off ${ }^{39,44}$.

Interestingly, one meiotic process, which has not been directly linked with piRNAs so far, is the meiotic silencing of unsynapsed chromosomes (MSUC). This mechanism is essential for meiosis, 
conserved across mammals, and results in transcriptional silencing of any chromosomes that are unpaired at the pachytene stage. One of the best studied examples for this is the meiotic silencing of the sex chromosomes in male germ cells due to their largely unpaired nature ${ }^{88}$. Studies have shown that conditional knockout of MILI after birth, therefore avoiding adverse phenotypes from MILI's prenatal role, has no impact on the establishment of meiotic silencing across the $\mathrm{X}$ and $\mathrm{Y}$ chromosomes ${ }^{89}$. Interestingly, while piRNA clusters are absent from the $\mathrm{X}$ chromosome in the mouse, profiling of the piRNA repertoire in marmoset identified several piRNA cluster on the $\mathrm{X}$ chromosome, suggesting that these regions may escape meiotic silencing ${ }^{81}$.

\section{Additional mechanisms for transposon control}

Periods of global epigenetic remodeling present TEs with an opportunity to become activated and retrotranspose, generating the need for the piRNA pathway in the male germline. Epigenetic reprogramming, however, is not unique to the male germline but also an essential process in female gametogenesis as well as preimplantation development in mouse embryos. Yet, the piRNA pathway is dispensable both in mouse oocytes as well as early embryos despite frequent upregulation of $\mathrm{TEs}^{90-92}$.

A possible explanation for the independence of the female germline in mice could be the lack of the fourth PIWI family member that exists in other mammals. PIWIL3 is found across eutherian mammals but is missing in the Muridae family comprising mice and rats. While its function remains largely elusive, it might hold some female germline-specific function as indicated by its exclusive expression in bovine oocytes ${ }^{93}$. In addition to PIWIL3, recent studies also found PIWIL1 expressed in both bovine oocytes and human fetal ovaries and suggest a role for piRNAs in the female germline of other mammals ${ }^{49,95}$. A possible compensation for the loss of PIWIL3 in the Muridae family is the expression of an oocyte-specific Dicer isoform (DicerO) originating from a retrotransposon insertion. This insertion drives the expression of a shortened Dicer variant that is more efficient in processing double-stranded RNA into endogenous siRNAs ${ }^{96}$. In general, it has been shown that oogenesis is far more dependent on Dicer and other small RNA biogenesis factors ${ }^{40,97}$. However, small RNAs, such as microRNAs, appear to function unperturbed throughout spermatogenesis. They are also not required for oogenesis and are in fact drastically downregulated during oocyte maturation, potentially to avoid competing effects between microRNA and endo-siRNA binding to AGO proteins ${ }^{98,99}$.

Apart from germ cell development, early embryonic development is another developmental window during which the regulation of transposable elements is vital for proper cell differentiation ${ }^{91}, 92$. While some studies have implicated low levels of piRNA expression in embryonic stem cells (ESCs) ${ }^{100}$, their presence does not appear to be crucial. However, it has been shown that ESCs and early embryos show high levels of repeat activation and while the activation of repetitive elements is often part of the developmental program and helps to orchestrate gene expression, a more sophisticated fine-tuned system is required to ensure proper regulation ${ }^{101-104}$. TE silencing in early embryos mostly relies on transcriptional gene silencing mediated by H3K9me3 instead of DNA methylation ${ }^{105-107}$. One mechanism to specifically target transcriptional gene silencing to TEs is based on the large family of Cys2-His2 zinc finger (C2H2-ZF) proteins that form the largest family of transcription factors in both the mouse and human genome ${ }^{108}$. DNA binding is mediated via the use of up to several dozen $\mathrm{C} 2 \mathrm{H} 2$ - $\mathrm{ZF}$ domains in a combinatorial manner and results in large binding motifs. These proteins are often coupled to effector domains, the most common being the Krüppel-associated box (KRAB), and upon binding are called KRAB-ZFPs in mice or KZNFs in humans ${ }^{109}$. KRAB-ZFPs mediate transcriptional silencing by interacting with the corepressor protein KAP1 (KRAB-associated protein 1, also known as TRIM28), which serves as a scaffold domain to recruit chromatin modifiers like the histone lysine-methyl transferase SETDB1 ${ }^{110}$.

KRAB-ZFPs are rapidly evolving and can be correlated to the emergence of species-specific TEs ${ }^{11}$. Indeed, an elegant study using transchromosomic mouse ESCs that carry one human chromosome was able to identify two human KZNFs that are necessary to repress two retrotransposon families that emerged relatively recently during human genome evolution ${ }^{112}$. Over time, repressed TEs will accumulate a sufficient number of mutations to render them inactive, making the KRAB-ZFP involvement in their recognition obsolete, which is reflected by the large number of KRAB-ZFP pseudogenes in the genome ${ }^{111}$. To date, only a few KRAB-ZFPs have been linked to a specific repeat element and their highly species-specific activities prohibit to draw conclusions from other model organisms. Assigning specific functions to KRAB-ZFPs in different species will remain challenging but the recent advance in identifying binding motifs and genomic target sites for numerous mouse and human KRAB-ZFPs will help to shed light onto this matter ${ }^{113-116}$. The KRAB-KAP1 pathway is an elegant example of co-adaptation between host genomes and their specific repeat content, which is dependent on gene duplication and mutation and therefore relatively slow. This could give highly active TEs the opportunity to cause major genomic damage until adaptation occurs. Our current understanding on how this adaptation is driven or directed is still very limited.

\section{Future challenges}

Many parts and factors of the piRNA biogenesis pathway in mammals still remain unknown due to the lack of easy-to-use in vitro models to study this pathway. Recently published protocols for the differentiation of ESCs into PGCs or further into sperm $^{117,118}$ might allow the study of mammalian piRNAs in more detail. As of to date, most studies rely on the use of mouse knockout models and candidate genes are often chosen based on their described function in other organisms, such as Drosophila. However, piRNA pathway components identified in Drosophila are not always conserved in mammals, such as the rhinodeadlock-cutoff complex ${ }^{119}$, therefore making the mechanistic comparisons from insect to mammalian systems challenging. Without knowing the exact piRNA biogenesis pathway, other aspects such as identifying the signal that directs piRNA precursors to be processed, similar to piRNA trigger sequences in Drosophila $^{75}$, remain a challenge.

\section{Conclusions}

While silencing of TEs in the germline is the unifying function of piRNAs observed across all animals, piRNAs have evolved and adapted additional functions in mammalian spermatogenesis. This includes the establishment of proper DNA methylation including paternal imprints as well as regulating meiotic crossovers by defining the chromatin state. Furthermore, piRNAs have recently been shown to be essential for proper spermatogenesis by controlling and orchestrating the transcriptional program via the regulation of spermatogenic mRNAs on both transcriptional and post-transcriptional levels.

Received: 5 April 2017 Accepted: 14 August 2017

Published online: 10 November 2017 


\section{References}

1. de Koning, A. P., Gu, W., Castoe, T. A., Batzer, M. A. \& Pollock, D. D. Repetitive elements may comprise over two-thirds of the human genome. PLoS Genet. 7, e1002384 (2011)

2. Slotkin, R. K. \& Martienssen, R. Transposable elements and the epigenetic regulation of the genome. Nat. Rev. Genet. 8, 272-285 (2007).

3. Levin, H. L. \& Moran, J. V. Dynamic interactions between transposable elements and their hosts. Nat. Rev. Genet. 12, 615-627 (2011).

4. Surani, M. A. \& Hajkova, P. Epigenetic reprogramming of mouse germ cells toward totipotency. Cold Spring Harb. Symp. Quant. Biol. 75, 211-218 (2010).

5. Shi, X., Seluanov, A. \& Gorbunova, V. Cell divisions are required for L1 retrotransposition. Mol. Cell Biol. 27, 1264-1270 (2007).

6. Handel, M. A. \& Schimenti, J. C. Genetics of mammalian meiosis: regulation, dynamics and impact on fertility. Nat. Rev. Genet. 11, 124-136 (2010).

7. Seki, Y. et al. Cellular dynamics associated with the genome-wide epigenetic reprogramming in migrating primordial germ cells in mice. Development 134, 2627-2638 (2007).

8. Popp, C. et al. Genome-wide erasure of DNA methylation in mouse primordial germ cells is affected by AID deficiency. Nature 463, 1101-1105 (2010).

9. Tam, P. P. \& Snow, M. H. Proliferation and migration of primordial germ cells during compensatory growth in mouse embryos. J. Embryol. Exp. Morphol. 64, 133-147 (1981).

10. Kato, Y. et al. Role of the Dnmt3 family in de novo methylation of imprinted and repetitive sequences during male germ cell development in the mouse. Hum. Mol. Genet. 16, 2272-2280 (2007).

11. Bourc'his, D. \& Bestor, T. H. Meiotic catastrophe and retrotransposon reactivation in male germ cells lacking Dnmt3L. Nature 431, $96-99$ (2004).

This study shows that Dnmt $3 L$ deletion causes spermatogenic failure in mice due to DNA hypomethylation of genomic retrotransposons and their subsequent activation.

12. Ooi, S. K. T. et al. DNMT3L connects unmethylated lysine 4 of histone $\mathrm{H} 3$ to de novo methylation of DNA. Nature 448, 714-717 (2007).

13. Bellvé, A. R. et al. Spermatogenic cells of the prepuberal mouse. Isolation and morphological characterization. J. Cell Biol. 74, 68-85 (1977).

14. Baudat, F., Imai, Y. \& de Massy, B. Meiotic recombination in mammals: localization and regulation. Nat. Rev. Genet. 14, 794-806 (2013).

15. Keeney, S., Giroux, C. N. \& Kleckner, N. Meiosis-specific DNA double-strand breaks are catalyzed by Spo11, a member of a widely conserved protein family. Cell 88, 375-384 (1997).

16. Baudat, F. et al. PRDM9 is a major determinant of meiotic recombination hotspots in humans and mice. Science 327, 836-840 (2010).

17. Myers, S. et al. Drive against hotspot motifs in primates implicates the PRDM9 gene in meiotic recombination. Science 327, 876-879 (2010).

18. Parvanov, E. D., Petkov, P. M. \& Paigen, K. Prdm9 controls activation of mammalian recombination hotspots. Science 327, 835 (2010).

19. Zamudio, N. et al. DNA methylation restrains transposons from adopting a chromatin signature permissive for meiotic recombination. Genes Dev. 29, 1256-1270 (2015).

20. O'Donnell, L. Mechanisms of spermiogenesis and spermiation and how they are disturbed. Spermatogenesis 4, e979623 (2015).

21. Balhorn, R. The protamine family of sperm nuclear proteins. Genome Biol. 8 , 227 (2007).

22. Ernst, C. et al. Successful transmission and transcriptional deployment of a human chromosome via mouse male meiosis. eLife 5, e20235 (2016).

23. Soumillon, M. et al. Cellular source and mechanisms of high transcriptome complexity in the mammalian testis. Cell Rep. 3, 2179-2190 (2013).

24. Ward, M. C. et al. Latent regulatory potential of human-specific repetitive elements. Mol. Cell 49, 262-272 (2013).

25. Brykczynska, U. et al. Repressive and active histone methylation mark distinct promoters in human and mouse spermatozoa. Nat. Struct. Mol. Biol. 17, 679-687 (2010).

26. Erkek, S. et al. Molecular determinants of nucleosome retention at CpG-rich sequences in mouse spermatozoa. Nat. Struct. Mol. Biol. 20, 868-875 (2013).

27. Hammoud, S. S. et al. Distinctive chromatin in human sperm packages genes for embryo development. Nature 460, 473-478 (2009).

28. Carmell, M. A. et al. MIWI2 is essential for spermatogenesis and repression of transposons in the mouse male germline. Dev. Cell 12, 503-514 (2007).

29. Deng, W. \& Lin, H. miwi, a murine homolog of piwi, encodes a cytoplasmic protein essential for spermatogenesis. Dev. Cell 2, 819-830 (2002).

30. Kuramochi-Miyagawa, S. et al. Two mouse piwi-related genes: miwi and mili. Mech. Dev. 108, 121-133 (2001).

31. Aravin, A. et al. A novel class of small RNAs bind to MILI protein in mouse testes. Nature 442, 203-207 (2006).

32. Girard, A., Sachidanandam, R., Hannon, G. J. \& Carmell, M. A. A germlinespecific class of small RNAs binds mammalian Piwi proteins. Nature $\mathbf{4 4 2}$, 199-202 (2006)
33. Grivna, S. T., Beyret, E., Wang, Z. \& Lin, H. A novel class of small RNAs in mouse spermatogenic cells. Genes Dev. 20, 1709-1714 (2006).

34. Lau, N. C. et al. Characterization of the piRNA complex from rat testes. Science 313, 363-367 (2006).

Results in Referenes 31-34 independently demonstrate the existence of piRNAs as a new class of small RNAs with regulatory potential in the rodent germline.

35. Cerutti, L., Mian, N. \& Bateman, A. Domains in gene silencing and cell differentiation proteins: the novel PAZ domain and redefinition of the Piwi domain. Trends Biochem. Sci. 25, 481-482 (2000).

36. Song, J.-J., Smith, S. K., Hannon, G. J. \& Joshua-Tor, L. Crystal structure of argonaute and its implications for RISC slicer activity. Science 305, 1434-1437 (2004).

37. Ma, J.-B. et al. Structural basis for $5^{\prime}$-end-specific recognition of guide RNA by the A. fulgidus Piwi protein. Nature 434, 666-670 (2005).

38. Parker, J. S., Roe, S. M. \& Barford, D. Structural insights into mRNA recognition from a PIWI domain-siRNA guide complex. Nature 434, 663-666 (2005).

39. Vourekas, A. et al. Mili and Miwi target RNA repertoire reveals piRNA biogenesis and function of Miwi in spermiogenesis. Nat. Struct. Mol. Biol. 19, 773-781 (2012).

40. Tam, O. H. et al. Pseudogene-derived small interfering RNAs regulate gene expression in mouse oocytes. Nature 453, 534-538 (2008).

41. Watanabe, T. et al. Endogenous siRNAs from naturally formed dsRNAs regulate transcripts in mouse oocytes. Nature 453, 539-543 (2008).

42. Nandi, S. et al. Roles for small noncoding RNAs in silencing of retrotransposons in the mammalian brain. Proc. Natl Acad. Sci. USA 113, 12697-12702 (2016).

43. Ng, K. W. et al. Piwi-interacting RNAs in cancer: emerging functions and clinical utility. Mol. Cancer 15, 5 (2016).

44. Castañeda, J. et al. Reduced pachytene piRNAs and translation underlie spermiogenic arrest in Maelstrom mutant mice. EMBO J. 33, 1999-2019 (2014).

45. Vourekas, A. et al. The RNA helicase MOV10L1 binds piRNA precursors to initiate piRNA processing. Genes Dev. 29, 617-629 (2015).

46. Zheng, K. et al. Mouse MOV10L1 associates with Piwi proteins and is an essential component of the Piwi-interacting RNA (piRNA) pathway. Proc. Nat Acad. Sci. USA 107, 11841-11846 (2010).

47. Ipsaro, J. J., Haase, A. D., Knott, S. R., Joshua-Tor, L. \& Hannon, G. J. The structural biochemistry of Zucchini implicates it as a nuclease in piRNA biogenesis. Nature 491, 279-283 (2012).

48. Nishimasu, H. et al. Structure and function of Zucchini endoribonuclease in piRNA biogenesis. Nature 491, 284-287 (2012).

49. Cora, E. et al. The MID-PIWI module of Piwi proteins specifies nucleotide- and strand-biases of piRNAs. RNA 20, 773-781 (2014)

50. Saxe, J. P., Chen, M., Zhao, H. \& Lin, H. Tdrkh is essential for spermatogenesis and participates in primary piRNA biogenesis in the germline. EMBO J. 32, 1869-1885 (2013).

51. Kawaoka, S., Izumi, N., Katsuma, S. \& Tomari, Y. $3^{\prime}$ end formation of PIWIinteracting RNAs in vitro. Mol. Cell 43, 1015-1022 (2011).

52. Aravin, A. A., Sachidanandam, R., Girard, A., Fejes-Toth, K. \& Hannon, G. J. Developmentally regulated piRNA clusters implicate MILI in transposon control. Science 316, 744-747 (2007).

53. Aravin, A. A. et al. A piRNA pathway primed by individual transposons is linked to de novo DNA methylation in mice. Mol. Cell 31, 785-799 (2008). This study dissects the spatio-temporal expression of murine Piwi proteins during spermatogenesis and links the piRNA machinery to DNA methylation of transposable elements.

54. Lim, S. L. et al. HENMT1 and piRNA stability are required for adult male germ cell transposon repression and to define the spermatogenic program in the mouse. PLoS Genet. 11, e1005620 (2015).

55. Wang, Y. et al. Nucleation, propagation and cleavage of target RNAs in Ago silencing complexes. Nature 461, 754-761 (2009).

56 . Wang, W. et al. The initial uridine of primary piRNAs does not create the tenth adenine that is the hallmark of secondary piRNAs. Mol. Cell 56, 708-716 (2014).

57. De Fazio, S. et al. The endonuclease activity of Mili fuels piRNA amplification that silences LINE1 elements. Nature 480, 259-263 (2011).

58. Wasik, K. A. et al. RNF17 blocks promiscuous activity of PIWI proteins in mouse testes. Genes Dev. 29, 1403-1415 (2015).

59. Yang, Z. et al. PIWI slicing and EXD1 drive biogenesis of nuclear piRNAs from cytosolic targets of the mouse piRNA pathway. Mol. Cell 61, 138-152 (2016).

60. Di Giacomo, M. et al. Multiple epigenetic mechanisms and the piRNA pathway enforce LINE1 silencing during adult spermatogenesis. Mol. Cell 50, 601-608 (2013).

This work characterizes how DNA methylation, repressive histone modifications and the piRNA pathway act together to enforce repression of transposable elements during adult spermatogenesis. 
61. Kuramochi-Miyagawa, S. et al. Mili, a mammalian member of piwi family gene, is essential for spermatogenesis. Dev. Camb. Engl. 131, 839-849 (2004).

62. Kuramochi-Miyagawa, S. et al. DNA methylation of retrotransposon genes is regulated by Piwi family members MILI and MIWI2 in murine fetal testes. Genes Dev. 22, 908-917 (2008).

63. Aravin, A. A. et al. Cytoplasmic compartmentalization of the fetal piRNA pathway in mice. PLoS Genet. 5, e1000764 (2009).

64. Molaro, A. et al. Two waves of de novo methylation during mouse germ cell development. Genes Dev. 28, 1544-1549 (2014).

65 . Itou, D. et al. Induction of DNA methylation by artificial piRNA production in male germ cells. Curr. Biol. 25, 901-906 (2015).

66. Pezic, D., Manakov, S. A., Sachidanandam, R. \& Aravin, A. A. piRNA pathway targets active LINE1 elements to establish the repressive $\mathrm{H} 3 \mathrm{~K} 9 \mathrm{me} 3$ mark in germ cells. Genes Dev. 28, 1410-1428 (2014).

67. Manakov, S. A. et al. MIWI2 and MILI have differential effects on piRNA biogenesis and DNA methylation. Cell Rep. 12, 1234-1243 (2015).

68. Nagamori, I. et al. Comprehensive DNA methylation analysis of retrotransposons in male germ cells. Cell Rep. 12, 1541-1547 (2015).

69. Vasiliauskaite, L. et al. A MILI-independent piRNA biogenesis pathway empowers partial germline reprogramming. Nat. Struct. Mol. Biol. 24, 604-606 (2017).

70. Tachibana, M., Nozaki, M., Takeda, N. \& Shinkai, Y. Functional dynamics of H3K9 methylation during meiotic prophase progression. EMBO J. 26, 3346-3359 (2007)

71. Watanabe, T. et al. Role for piRNAs and noncoding RNA in de novo DNA methylation of the imprinted mouse Rasgrf1 locus. Science 332, 848-852 (2011).

72. Robine, N. et al. A broadly conserved pathway generates $3^{\prime}$ UTR-directed primary piRNAs. Curr. Biol. 19, 2066-2076 (2009).

73. Chirn, G. et al. Conserved piRNA expression from a distinct set of piRNA cluster loci in eutherian mammals. PLoS Genet. 11, e1005652 (2015).

74. Li, X. Z. et al. An ancient transcription factor initiates the burst of piRNA production during early meiosis in mouse testes. Mol. Cell 50, 67-81 (2013).

75. Homolka, D. et al. PIWI slicing and RNA elements in precursors instruct directional primary piRNA biogenesis. Cell Rep 12, 418-428 (2015).

76. Tanaka, T. et al. Tudor domain containing 7 (Tdrd7) is essential for dynamic ribonucleoprotein (RNP) remodeling of chromatoid bodies during spermatogenesis. Proc. Natl Acad. Sci. USA 108, 10579-10584 (2011).

77. Vagin, V. V. et al. Proteomic analysis of murine piwi proteins reveals a role for arginine methylation in specifying interaction with tudor family members. Genes Dev. 23, 1749-1762 (2009).

78. Chinwalla, A. T. et al. Initial sequencing and comparative analysis of the mouse genome. Nature 420, 520-562 (2002).

79. Reuter, M. et al. Miwi catalysis is required for piRNA amplificationindependent LINE1 transposon silencing. Nature 480, 264-267 (2011) This study demonstrates that even in mouse adult spermatogenesis, MIWI is still essential for transposon silencing.

80. Muerdter, F. et al. Production of artificial piRNAs in flies and mice. RNA 18, 42-52 (2012).

81. Hirano, T. et al. Small RNA profiling and characterization of piRNA clusters in the adult testes of the common marmoset, a model primate. RNA 20, 1223-1237 (2014)

82. Ronsseray, S., Lehmann, M. \& Anxolabéhère, D. The maternally inherited regulation of $\mathrm{P}$ elements in Drosophila melanogaster can be elicited by two $\mathrm{P}$ copies at cytological site $1 \mathrm{~A}$ on the X chromosome. Genetics 129, 501-512 (1991).

83. Kimmins, S. \& Sassone-Corsi, P. Chromatin remodelling and epigenetic features of germ cells. Nature 434, 583-589 (2005).

84. Watanabe, T., Cheng, E., Zhong, M. \& Lin, H. Retrotransposons and pseudogenes regulate mRNAs and lncRNAs via the piRNA pathway in the germline. Genome Res. 25, 368-380 (2015).

85. Zhang, P. et al. MIWI and piRNA-mediated cleavage of messenger RNAs in mouse testes. Cell Res. 25, 193-207 (2015).

86. Goh, W. S. S. et al. piRNA-directed cleavage of meiotic transcripts regulates spermatogenesis. Genes Dev. 29, 1032-1044 (2015).

87. Gou, L.-T. et al. Pachytene piRNAs instruct massive mRNA elimination during late spermiogenesis. Cell Res. 24, 680-700 (2014).

88. Turner, J. M. A. Meiotic silencing in mammals. Annu. Rev. Genet. 49, 395-412 (2015).

89. Beyret, E. \& Lin, H. Pinpointing the expression of piRNAs and function of the PIWI protein subfamily during spermatogenesis in the mouse. Dev. Biol. 355, 215-226 (2011).
90. Fadloun, A. et al. Chromatin signatures and retrotransposon profiling in mouse embryos reveal regulation of LINE-1 by RNA. Nat. Struct. Mol. Biol. 20, 332-338 (2013).

91. Macfarlan, T. S. et al. Embryonic stem cell potency fluctuates with endogenous retrovirus activity. Nature 487, 57-63 (2012).

92. Peaston, A. E. et al. Retrotransposons regulate host genes in mouse oocytes and preimplantation embryos. Dev. Cell 7, 597-606 (2004).

The first evidence that transposable elements influence gene expression in oocytes and embryos by functioning as alternative promoters are provided in this report.

93. Roovers, E. F. et al. Piwi proteins and piRNAs in mammalian oocytes and early embryos. Cell Rep. 10, 2069-2082 (2015)

94. Russell, S. J. et al. Identification of PIWIL1 isoforms and their expression in bovine testes, oocytes, and early embryos. Biol. Reprod. 94, 75 (2016).

95 . Williams, Z. et al. Discovery and characterization of piRNAs in the human fetal ovary. Cell Rep. 13, 854-863 (2015).

96. Flemr, M. et al. A retrotransposon-driven dicer isoform directs endogenous small interfering RNA production in mouse oocytes. Cell 155, 807-816 (2013).

97. Murchison, E. P. et al. Critical roles for Dicer in the female germline. Genes Dev. 21, 682-693 (2007).

98. Ma, J. et al. MicroRNA activity is suppressed in mouse oocytes. Curr. Biol. 20 , 265-270 (2010).

99. Suh, N. et al. MicroRNA function is globally suppressed in mouse oocytes and early embryos. Curr. Biol. 20, 271-277 (2010).

100. Marchetto, M. C. N. et al. Differential L1 regulation in pluripotent stem cells of humans and apes. Nature 503, 525-529 (2013).

101. Franke, V. et al. Long terminal repeats power evolution of genes and gene expression programs in mammalian oocytes and zygotes. Genome Res. doi:10.1101/gr.216150.116 (2017).

102. Göke, J. et al. Dynamic transcription of distinct classes of endogenous retroviral elements marks specific populations of early human embryonic cells. Cell Stem Cell 16, 135-141 (2015).

103. Ishiuchi, T. et al. Early embryonic-like cells are induced by downregulating replication-dependent chromatin assembly. Nat. Struct. Mol. Biol. 22, 662-671 (2015).

104. Svoboda, P. et al. RNAi and expression of retrotransposons MuERV-L and IAP in preimplantation mouse embryos. Dev. Biol. 269, 276-285 (2004).

105. Karimi, M. M. et al. DNA methylation and SETDB1/H3K9me3 regulate predominantly distinct sets of genes, retroelements, and chimeric transcripts in mESCs. Cell Stem Cell 8, 676-687 (2011).

106. Matsui, T. et al. Proviral silencing in embryonic stem cells requires the histone methyltransferase ESET. Nature 464, 927-931 (2010).

107. Walter, M., Teissandier, A., Pérez-Palacios, R. \& Bourc'his, D. An epigenetic switch ensures transposon repression upon dynamic loss of DNA methylation in embryonic stem cells. eLife 5, e11418 (2016).

108. Emerson, R. O. \& Thomas, J. H. Adaptive evolution in zinc finger transcription factors. PLoS Genet. 5, e1000325 (2009).

109. Urrutia, R. KRAB-containing zinc-finger repressor proteins. Genome Biol. 4 231 (2003).

110. Schultz, D. C., Ayyanathan, K., Negorev, D., Maul, G. G. \& Rauscher, F. J. SETDB1: a novel KAP-1-associated histone H3, lysine 9-specific methyltransferase that contributes to HP1-mediated silencing of euchromatic genes by KRAB zinc-finger proteins. Genes Dev. 16, 919-932 (2002).

111. Thomas, J. H. \& Schneider, S. Coevolution of retroelements and tandem zinc finger genes. Genome Res. 21, 1800-1812 (2011)

112. Jacobs, F. M. J. et al. An evolutionary arms race between KRAB zinc-finger genes ZNF91/93 and SVA/L1 retrotransposons. Nature 516, 242-245 (2014).

By using a transchromosomic model, this report illustrates the co-evolution of recently evolved retrotransposons that are kept under control by lineage specific zinc-finger proteins and the retrotransposons' strategy to overcome repression.

113. Ecco, G. et al. Transposable elements and Their KRAB-ZFP controllers regulate gene expression in adult tissues. Dev. Cell 36, 611-623 (2016).

114. Imbeault, M., Helleboid, P.-Y. \& Trono, D. KRAB zinc-finger proteins contribute to the evolution of gene regulatory networks. Nature 543, 550-554 (2017).

115. Najafabadi, H. S. et al. $\mathrm{C} 2 \mathrm{H} 2$ zinc finger proteins greatly expand the human regulatory lexicon. Nat. Biotechnol. 33, 555-562 (2015).

116. Schmitges, F. W. et al. Multiparameter functional diversity of human $\mathrm{C} 2 \mathrm{H} 2$ zinc finger proteins. Genome Res. 26, 1742-1752 (2016).

117. von Meyenn, F. et al. Comparative principles of DNA methylation reprogramming during human and mouse in vitro primordial germ cell specification. Dev. Cell 39, 104-115 (2016). 
118. Zhou, Q. et al. Complete meiosis from embryonic stem cell-derived germ cells in vitro. Cell Stem Cell 18, 330-340 (2016).

119. Mohn, F., Sienski, G., Handler, D. \& Brennecke, J. The rhino-deadlock-cutoff complex licenses noncanonical transcription of dual-strand piRNA clusters in Drosophila. Cell 157, 1364-1379 (2014).

\section{Acknowledgements}

We are grateful for the support by Cancer Research UK (CE, DTO), European Research Council (DTO), Wellcome Trust (DTO), SciLifeLab Fellow Program (CK), Knut and Alice Wallenberg (CK) and Ruth and Richard Julin (CK).

\section{Author contributions}

CE, DTO, CK: drafting and/or revision of the manuscript.

\section{Additional information}

Competing interests: The authors declare no competing financial interests.

Reprints and permission information is available online at http://npg.nature.com/ reprintsandpermissions/
Publisher's note: Springer Nature remains neutral with regard to jurisdictional claims in published maps and institutional affiliations.

(c) (i) Open Access This article is licensed under a Creative Commons Attribution 4.0 International License, which permits use, sharing, adaptation, distribution and reproduction in any medium or format, as long as you give appropriate credit to the original author(s) and the source, provide a link to the Creative Commons license, and indicate if changes were made. The images or other third party material in this article are included in the article's Creative Commons license, unless indicated otherwise in a credit line to the material. If material is not included in the article's Creative Commons license and your intended use is not permitted by statutory regulation or exceeds the permitted use, you will need to obtain permission directly from the copyright holder. To view a copy of this license, visit http://creativecommons.org/ licenses/by/4.0/.

(C) The Author(s) 2017 